\title{
$\operatorname{Conf}-9310297-2$ \\ UCRLAC. $1 / 5928$ \\ PREPRINT
}

\section{Identification of cDNAs by direct hybridization using cosmid probes}

G. G. Lennon and K. Lieuallen

This paper was prepared for submittal to the Proceedings of the Third International Workshop in the Identification of Transcribed Sequences, New Orleans, LA, October 2-4, 1993

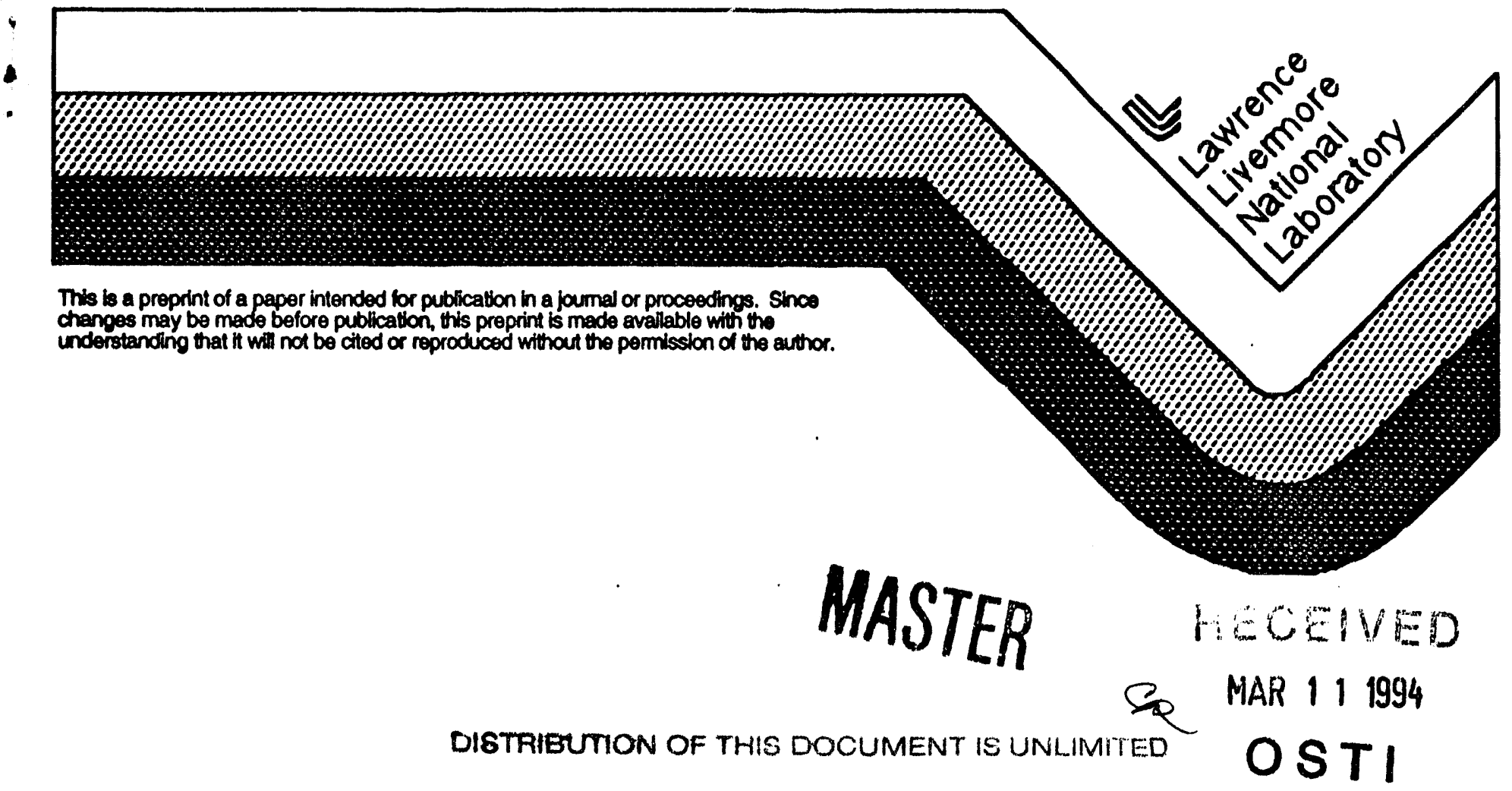




\section{DISCLAIMER}

This document was prepared as an account of work sponsored by an agency of the United States Government. Neither the United States Government nor the University of California nor any of their emplojees, makes any warranty, express or implied, or assumes any legal liability or responsibility for the accuracy, completeness, or usefulness of any information, apparatus, product, or process disclased, or represents that its use would not infringe privately owned rights. Reference herein to any specific commercial products, process, or service by trade name, trademark, manufacturer, or otherwise, does not necessarily constitute or imply its endorsement, recommendation, or favoring by the United States Government or the University of California. The views and opinions of authors expressed herein do not necessarily state or relect those of the United States Government or the University of Callfornis, and shall not be used for advertising or product endorsement purposes. 
Identification of cDNAs By Direct Hybridization Using Cosmid Probes

Gregory G. Lennon and Kimberly Lieuallen

Human Genome Center, L-452

Lawrence Livermore National Laboratory

Livermore, California 94550

\section{ABSTRACT}

The goal of this effort is to quickly obtain as many chromosome-specific cDNAs with as much map and sequence detail as possible. Many techniques have been proposed to isolate and identify genes within defined genomic regions; the technique discussed here is direct hybridization of a relatively complex genomic probe, an entire cosmid clone, to cDNA libraries. This method continues to be a straightforward technique with a fair number of successes.

\section{INTRODUCTION}

Many techniques are available to find cDNAs, and new techniques are continually being developed and streamlined. Methods include [1] expression-dependent (northerm blot analysis, cDNA library screening with complex probes, hybrid selection), expression-independent (cross-species sequence homology, CpG island selection, exontrapping, splice-site screening), sequence-based methods, subtractive cDNA hybridization, coincident sequence cloning, and hnRNA library screening.

Our goal is the isolation and characterization of genes (and their cognate cDNAs) on human chromosome 19. The euchromatic portion of human chromosome 19 is estimated to be $\mathbf{4 5}$ megabases, and to contain about 2000 genes. Therefore, 1 gene is estimated to be present every $22.5 \mathrm{~kb}$, or over 1 gene per $40 \mathrm{~kb}$ cosmid on average.

One of the most direct methods of obtaining these cDNAs, and the focus of this chapter, is the use of hybridization to directly identify cDNAs encoded within the genomic probes used. Cosmids generated from flow-sorted chromosome 19 libraries are used as probes against arayed CDNA libraries [2], and thus the cDNAs identified should be chromosome-specific. The aspects of this method to be discussed are: probes; targets; hybridization; analysis; and, verification. The discussion will conclude with an overview of the pro's and con's of this technique. Although this effort is focused on chromosome 19 , it is just as applicable to other chromosomes, and even to non-chromosome-specific efforts.

\section{MATERIALS AND METHODS}

\section{Probes}

The chromosome 19 specific cosmid libraries were made at Lawrence Livermore National Laboratory [3]. Currently, we have three libraries in Lawrist vectors. The average insert size of the cosmids is $40 \mathrm{~kb}$. In an attempt to increase the chances of identifying a gene we screened cosmids for SacIl sites, since SacII sites include: the dinucleotide CPG which may be indicative of nearby genes. Typically, cosmid DNA is purified using QIAGEN columns. Approximately 100ng of cosmid DNA is incubated with 4 units of Sac II enzyme at $37^{\circ} \mathrm{C}$ overnight. After electrophorectic analysis to 
determine the number of fragments, those cosmids with 3 or more Sac II sites are singled out for use as probes.

Approximately $25 \mathrm{ng}$ of cosmid DNA is random-labelled by nonamer priming including 32.P (Prime-It, Stratagene). The probe is purified from unincorporated 32.P using a G-50 column. After this purification process, we typically get $1 \times 10^{5} \mathrm{cpm}$ per microliter of probe. We used 3ug of pBluescript KS- DNA, 5ug of poly A, 10ug of human Cot-1 DNA and 50ug of salmon sperm DNA to block the probe. The probe is annealed with the blocking agents for approximately 30 minutes at $65^{\circ} \mathrm{C}$.

\section{Targets}

The normalized human infant brain cDNA library created by Dr. Bento Soares (unpublished) was the target in our hybridizations with cosmid probes. We arrayed approximately 40,000 cDNA clones from this library into 96-well dishes. From this arrayed library high density colony filters were generated. These filters are arranged such that there are 6,912 independent clones present in duplicate on each filter, for a total of 13,824 clones per filter. The ORCA (Hewlett-Packard) robot was used to spot the high density colony filters, spotting at a rate of approximately 40,000 clones per hour. After spotting, the colonies were grown overnight and the DNA fixed to the filter the next day.

\section{Hybridizations and Analyses}

The filters were typically pre-hybridized for 2.12 hours in a $2 \%$ SDS hybridization solution containing $0.6 \mathrm{M} \mathrm{NaCl}, 10 \mathrm{mM}$ EDTA, $50 \mathrm{mM}$ Tris (pH 7), $0.1 \%$ Na-pyrophosphate, $10 \%$ dextran sulfate (of a $50 \%$ stock solution), and $2 \%$ SDS. We blocked the filters during this pre-hybridization period with 500 ug of salmon sperm DNA, 3ug of pBluescript DNA, and 200ug of poly U. Both the pre-hybridization and hybridization are done at $65^{\circ} \mathrm{C}$. After the pre-hybridization period the probe is added and allowed to hybridize overnight. Typically, two low stringency washes and two high stringency washes are performed for 30 minutes each at $65^{\circ} \mathrm{C}$. The low stringency solution contains $2 \mathrm{X}$ SSC and $20 \%$ SDS, while the high stringency wash contains $0.2 \mathrm{X}$ SSC and 1\% SDS.

After the washes, the filters are exposed to PhosphorImager screens (Molecular Dynamics) overnight. After exposure the positives (present in duplicate) on the images are evaluated and called manually. Clone array position (plate and well) is calculated, and compared with other information available for those array positions. The filters themselves can be re-used approximately ten times. Typically, the filters are stripped for about 8 hours at room temperature with a solution of $2 \mathrm{mM}$ EDTA and $0.1 \%$ SDS.

\section{Verification}

The verification procedures (Figure 1) begin with the selection of colonies which are positive. Glycerol stocks of these clones are used to inoculate mini-preps. Plasmid DNA is harvested using a Promega Wizard mini prep kit and then the DNA is sequenced. Sequences are sent off to be searched against Genbank sequences using the programs Blastn and Blastx [4]. Most cDNAs have primers developed using the "Primer" program available from the Whitehead Institute. The primers are then tested by PCR against total genomic DNA and against a hamster/human somatic cell hybid line containing human chromosome 19. Those cDNAs that show a product in the somatic cell hybrid line (but not in the hamster only parental cell line) are then used in a hybridization against high density colony filters containing clones from the Lawrence Livermore National Laboratory chromosome 19 cosmid library (as previously mentioned) and one northern blot with mRNA from human heart, brain, placenta, lung, liver, skeletal muscle, kidney, 
and pancreas tissue. The cosmids identified as positives are then verified by digesting them with EcoRI and blotting to a nylon membrane whereby this membrane is probed with the cDNA. Alternatively, DNA from identified cosmids is used as template in a $P C R$ reaction using the primers generated from the $c D N A$.

Figure 1. Flowchart of Verification Procedures.

A CDNA is only considered to be verified as originating from chromosome 19 if all three conditions are met, i.e. it maps by PCR to chromosome 19 , cosmids from the chromosome-specific cosmid library are isolated, and these cosmids are confirmed by PCR or hybridization to contain homologous sequences. Once a CDNA has been verified, further work on other cDNAs identified in the same original hybridization is suspended unless there is independent evidence supporting the existence of another gene encoded within the same cosmid. 


\section{RESULTS}

34 hybridizations have been performed using cosmids as probes on the normalized infant brain cDNA library ; a reasonably typical example of a good hybridization is shown in Figure 2. These 34 hybridizations have resulted in 330 positives, for an average of 9.7 clones per probe. About half of the hybridizations yield no positives, so the average number of positive clones per positive probe is about 20.

Figure 2. Hybridization Result. Total cosmid DNA from cosmid 29297 was used as probe; a portion of one filter, containing 3,360 independent cDNA clones (duplicated, upper and lower), is shown. In addition to the one positive, the reference clones present in the upper left corner of each $6 \times 6$ array unit are clearly visible.

Sequence has been derived from approximately $67 \%$ of the positive cDNAs. Sequence analysis reveals that even with blocking of both the probe and the filters during hybridization, approximately 5\% of the positives contain an Alu element. These clones are not analysed further. Another $1 \%$ of the positives are known genes from other chromosomes. The remaining $94 \%$ of sequenced positives lack significant homologies with known sequences.

Currently, primer pairs for $33 \mathrm{cDNAs}$ await testing, and others are in various stages of the verification process. On a per cosmid basis (and thus per hybridization) results for clones that have been completely analysed indicate that about $50 \%$ of hybridizations giving at least one positive yield a CDNA that van be verified to be from chromosome 19. Thus far, twelve new chromosome $19 \mathrm{cDNAs}$ have been identified. It 
is difficult to estimate the success rate on a per cDNA clone basis (rather than per hybridization) since analysis stops on most $\mathrm{cDNA}$ clones once one clone from the same hybridization has been verified as from chromosome 19.

Over 800 cosmids have been digested with SaclI, and of these, 29 had 3 or more SacII sites. This is in in keeping with studies on Sacll site frequency for other chromosomes [5]. Surprisingly, we have not seen a significant difference between 17 SacII-rich and 17 randomly chosen cosmids in the percentage of hybridizations that succeed in identifying a cDNA eventually verified to be from chromosome 19.

\section{DISCUSSION}

With this method we have identified and fine-mapped 12 independent anonymous cDNAs on chromosome 19 in approximately one person-year. Interestingly, this number (12) is also the total number of chromosome $19 \mathrm{cDNAs}$ assigned over the last year by four groups $[6,7,8,9]$ mapping cDNAs (from EST sequencing projects) using somatic cell hybrids. These latter cDNAs are initially mapped to chromosome resolution. By contrast, the cDNAs identified by the direct hybridization approach are mapped to a single restriction band of usually less than $10 \mathrm{~kb}$. As the physical map becomes more precise, the precision of the location of these cDNAs also increases since the CDNA is linked to an element of the physical map.

The positive aspects of the direct hybridization technique therefore include fine resolution mapping, and the use of straightforward and robust laboratory techniques. In contrast to methods involving PCR amplification, CDNA clones isolated are of the average length of the cDNA library, and thus significantly overlkb in length. The two main disadvantages involve the yield of cDNAs. First, about half of all hybridizations result in zero positives. Second, although $50 \%$ of the remaining hybridizations will yield a verifiable chromosome $19 \mathrm{cDNA}$, the amount of work required to sift the false positives out from the true positives is high, limiting the throughput of this approach.

We have found that the number of SacIl sites within a cosmid is not necessarily indicative of increased success with the direct hybridization approach; we have not found a higher true positive rate for the SacII rich cosmids compared with randomly selected cosmids. Because of this and the time it takes to screen cosmids for SacII, we no longer choose cosmid probes based on their SacII-site richness. Note, however, that chromosome 19 is a GC rich (and gene-rich) chromosome, and that screening for $\mathrm{CpG}$ rich cosmids may be of greater aid in identifying candidate probe regions on chromosomes that are GC poorer in general than chromosome 19.

In the future, we hope to apply this technique in screening arrayed full-length cDNA libraries. Currently, in order to isolate full-length versions of our new chromosome 19 cDNAs we usually screen a number of cDNA libraries, and check clone insert sizes against the mRNA size as estimated by the Northern blots. For numerous reasons, both for our and other groups, a great savings in time would be achieved if arrayed full-length libraries were available. Since many genes are not ubiquitously expressed, we are also planning on arraying high quality cDNA libraries from other tissues. The use of arrayed DNA spots (from either PCR or automated DNA mini-preps) for these or our current libraries would also give better results. Other improvements for this technique include the promise of automated image analysis programs to reliably call positives from the digitized images.

Although cDNAs have been isolated from phage cDNA libraries using probes as complex as YACs of size 270kb [10], and thus the equivalent of about 6 cosmids, it is likely that the practical limits on complexity are somewhere in between 40 and $250 \mathrm{~kb}$ for routine screening of cDNA colony filters. The use of pooled genomic probes that are of

this range of complexity may therefore be appropriate. These include microdissected material, pooled exon trap products, hybrid selection products, CpG islands isolated from 
flow-sorted chromosomes, or PCR prafucts from chromosome specific material using consensus motif primers (such as splice site sequences). For example, if there are 2,000 genes on chromosome 19, every gene could contribute up to approximately $100 \mathrm{bp}$ of sequence on average to a "whole chromosome" probe and the estimated complexity would still be within an acceptable range for routine screening of colony cDNA libraries.

\section{CONCLUSIONS}

The identification of cDNAs by direct hybridization of complex genomic probes continues to be of great utility. Using the arrayed normalized human infant brain cDNA library, we have achieved a high rate of discovery of novel chromosome $19 \mathrm{cDNAs}$. The keys to our success to date rely on at least two factors : first, a high quality cDNA library that is arrayed and thus available for reproducible screening by filter hybridization, and second, a rigorous verification process. Disadvantages of the technique include a high false positive rate, and thus a labor-intensive verification process. Future improvements aimed at reducing the false positive rate and increasing throughput include the use of fulllength cDNA libraries, the spotting of DNA rather than colonies, and the use of pooled genomic probes and automated image analysis.

\section{ACKNOWLEDGEMENTS}

This work was performed under the auspices of the U.S. Department of Energy by Lawrence Livermore National Laboratory under contract no. W-7405-Eng-48.

\section{REFERENCES}

1. J.E. Parrish and D.L. Nelson, Methods for finding genes: a major rate-limiting step in positional cloning, Gene Anal. and Tech.:10:29 (1993)

2. G.G. Lennon and H. Lehrach, Hybridization analyses of arrayed CDNA libraries, Trends in Genetics: 10: 314 (1991)

3. A.V. Carrano, P.J. de Jong, E. Branscomb, et al., Constructing chromosome- and region- specific cosmid maps of the human genome, Genome:31:1059 (1989)

4. S.F. Altschul, W. Gish, W. Miller, et al., Basic local alignment search tool, J. Mol. Biol.:215:403 (1990)

5. W.C. Golembieski, S.C. Smith, F. Recchia, et al., Isolation of large numbers of chromosome-3 specific cusmids containing clusters of rare restriction-endonuclease sites, Am. J. Hum. Gen.:49:581 (1991)

6. K. Okubo, N. Hori, R. Matoba et al., Large scale cDNA sequencing for analysis of quantitative and qualitative aspects of gene expression, Nature Genetics:2:173 (1992)

7. A.S. Durkin, D.R. Maglott, and W.C. Nierman, Chromosomal assignment of 38 human brain expressed sequence tags (ESTs) by analyzing fluorescently labeled PCR products from hybrid cell lines, Genomics:14:808 (1992)

8. M.H. Polymeropoulos, H. Xiao, A. Glodek, et al., Chromosomal assignment of 46 brain cDNAs, Genomics:12:492 (1992) 
9. L. Gieser and A. Swaroop, Expressed sequence tags and chromosomal localization of cDNA clones from a subtracted retinal pigment epithelium library, Genomics:13:873 (1992)

10. M.R. Wallace, D.A. Marchuk, L.B. Andersen et al., Type 1 neurofibromatosis: identification of a large transcript disrupted in thrse NF1 patients, Science:249:181 (1990) 


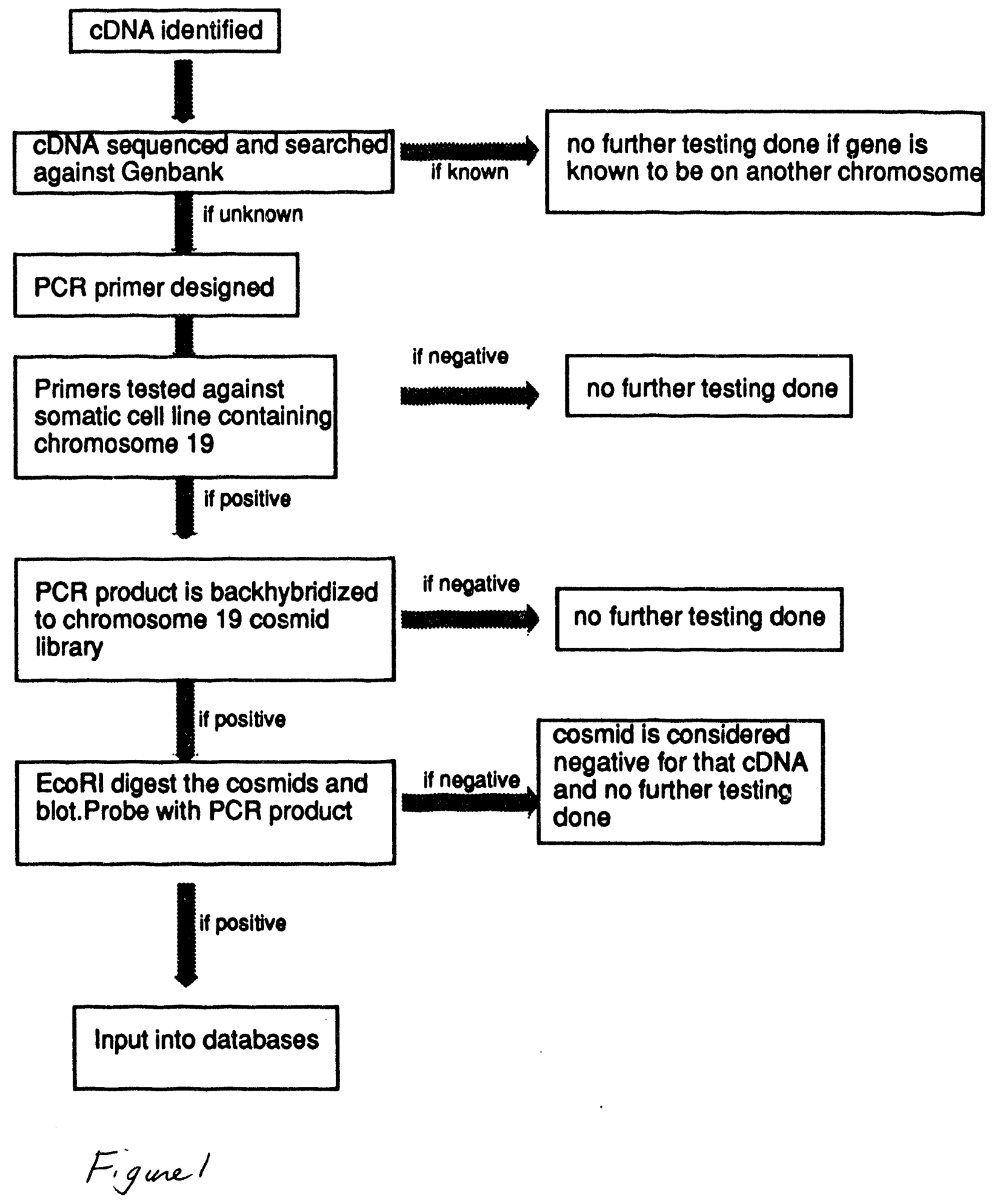




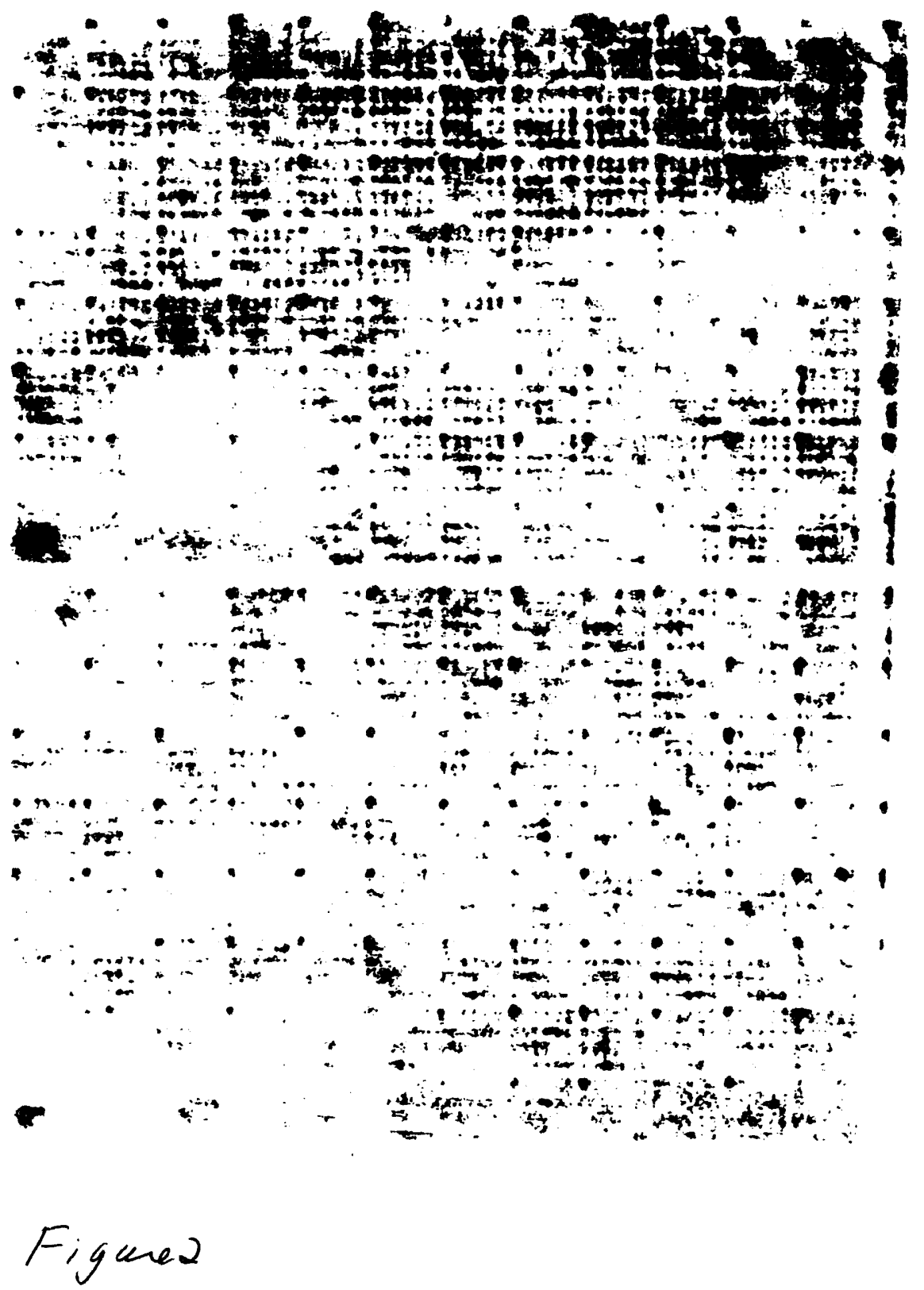



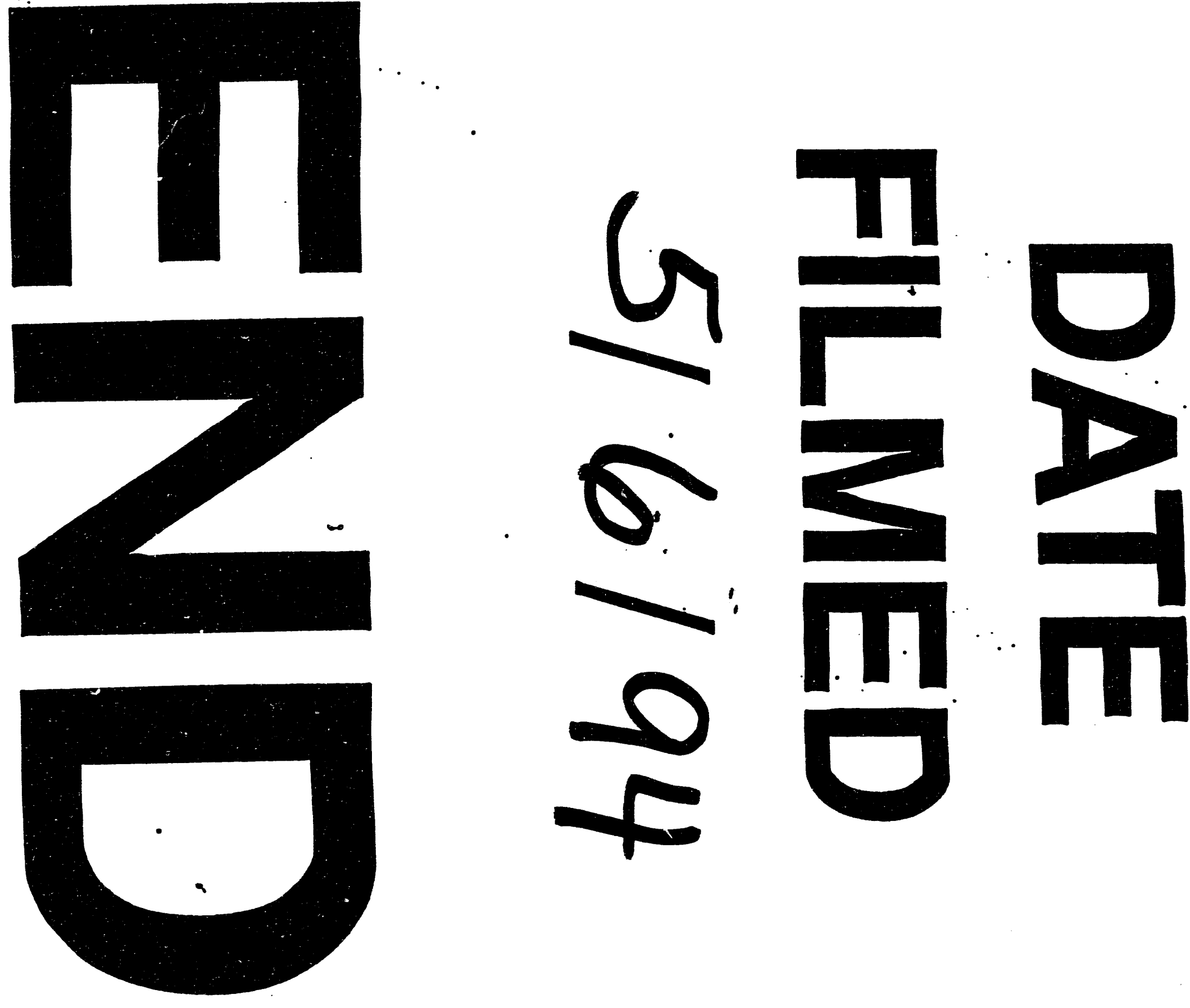

II 